\title{
Parents of two-phase flow and theory of "gas-lift"
}

\author{
Pavel Zitek ${ }^{1 \mathrm{a}}$ and Vaclav Valenta ${ }^{1}$ \\ ${ }^{1}$ UWB in Pilsen, Department of Power System Engineering, Univerzitni 8, 30614 Pilsen, Czech Republic
}

\begin{abstract}
This paper gives a brief overview of types of two-phase flow. Subsequently, it deals with their mutual division and problems with accuracy boundaries among particular types. It also shows the case of water flow through a pipe with external heating and the gradual origination of all kinds of flow. We have met it in solution of safety condition of various stages in pressurized and boiling water reactors. In the MSR there is a problem in the solution of gas-lift using helium as a gas and its secondary usage for clearing of the fuel mixture from gaseous fission products. Theory of gas-lift is described.
\end{abstract}

\section{Boiling Heat Transfer}

Suppose we first consider a heating element submerged in a pool of stationary fluid. A sketch of the heat flux transferred from the heating surface to the fluid versus the temperature difference between the surface and the bulk fluid temperature is shown in figure 1. From this sketch it is evident that one can distinguish several different regions of heat transfer.

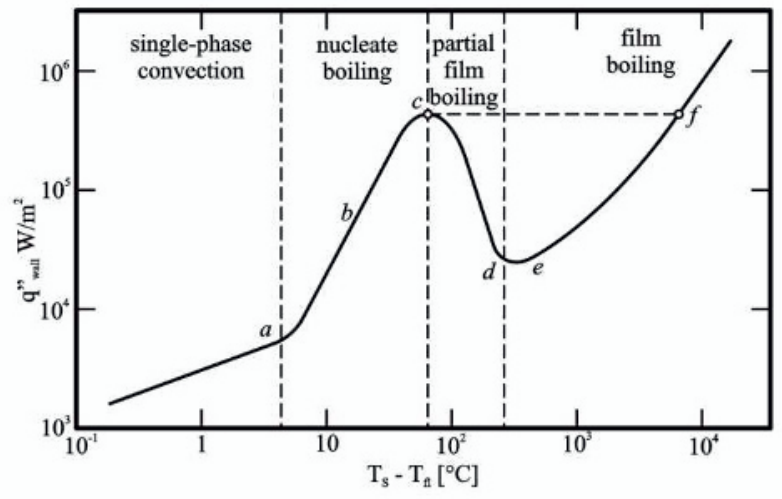

Figure 1 Heat flux versus temperature difference for poolboiling heat transfer

0 - a: There is little liquid superheat; heat transfer is by natural convection.

a - b: A few bubbles are formed but collapse after leaving surface (although agitation will increase heat transfer).

a-c: The number of bubbles formed increases rapidly - this is nucleate boiling regime.

c: $\quad$ "Burnout" point or DNB is reached.

$\mathrm{c}-\mathrm{d}$ : Bubbles become so numerous that they begin to coalesce and clump near the heating surface (the vapor covering the surface acts as a heat insulator) - this is known as partial nucleate boiling. d - e: A continuous blanket of vaper forms over the heating surface - film boiling regime.

e - f: Thermal radiation from the surface comes into play - film and radiation regime.

It should particularly be noted that if the heat flux $q^{\prime \prime}$ is the independent variable, then increasing $q$ " beyond the DNB point will result in a large increase in the surface temperature for a given coolant temperature (see figure 1). This phenomenon is known as burnout and must be avoided in reactor operation.

These concepts also apply in a flowing channel. Suppose we consider a channel with a uniform wall heat flux $q^{\prime \prime}{ }_{\text {wall }}$. Then one will find temperature profiles up the channel as shown in figure 2 . Once again we can distinguish several regions of heat transfer:

1. Region A: Single-phase convection.

In this region we can use the analysis we developed in the previous section to determine the convective heattransfer coefficient $h_{s}$ arising in Newton's law of cooling

$$
q^{\prime \prime}=h_{s} \cdot\left(T_{s}-T_{f l}\right)
$$

2. Region B: Subcooled boiling: $T_{S} \geq T_{\text {sat }}$ but $T_{\text {fl }}<T_{\text {sat }}$.

Once the clad surface temperature reaches the saturation temperature of the coolant, the temperature drop is no longer linear in the heat flux. In this region, the heat transfer process is extremely efficient, and the temperature difference is quite small compared to the other temperature differences in the core. In LWRs it is customary to use an empirical correlation developed [19] in order to calculate the temperature difference between the clad surface and the coolant:

$$
\left.\Delta T\right|_{\mathrm{COOL}}=T_{S}-T_{f l}=45 \exp \left(-\frac{p}{62}\right) \cdot\left(q^{\prime \prime}\right)^{0.25}
$$

where $p$ is the pressure in units of bar and $q$ " is the heat flux in units in $\mathrm{MW} / \mathrm{m}^{2}$. This correlation is valid for

\footnotetext{
${ }^{\mathrm{a}}$ Corresponding author: zitek@kke.zcu.cz
} 
pressures, temperatures, and coolant flow rates characteristic of both PWRs and BWRs. For example, in a PWR at 155 bar with a heat flux of $1.5 \cdot 10^{6} \mathrm{~W} / \mathrm{m}^{2}$, one would find a temperature difference of only $3{ }^{\circ} \mathrm{C}$. There will be very little change in the temperature difference with heat flux in this boiling regime. Once the clad surface temperature of the coolant, it tends to remain near that level unless the heat flux reaches the DNB value.

One can use these relationships to determine the position $z_{S C B}$ at which subcooled boiling begins. We equate $T_{f l}=T_{\text {sat }}$ in (2):

$$
T_{S}-T_{\text {sat }}=45 \exp \left(-\frac{p}{62}\right) \cdot\left(q^{\prime \prime}\right)^{0.25}
$$

Then noting also that

$$
q^{\prime \prime}=h_{s} \cdot\left(T_{S}-F_{f l}\right)
$$

We can find

$$
\begin{gathered}
\left.T_{f l}\right|_{z_{S C B}}=T_{S}-\frac{q^{\prime \prime}}{h} \\
\left.T_{f l}\right|_{z_{S C B}}=T_{s a t}+45 \exp \left(-\frac{p}{62}\right) \cdot\left(q^{\prime \prime}\right)^{0.25}-\frac{q^{\prime \prime}}{h_{s}}
\end{gathered}
$$

and then solve this for the position $z_{S C B}$.

3. Region C: Saturated or bulk boiling: $T_{f l}=T_{\text {sat }}$.

Eventually sufficient heat is transferred to the coolant that it reaches its saturation temperature and begins bulk boiling. Subsequent heat addition will cause further boiling, and hence the coolant temperature will remain essentially constant and equal to $T_{\text {sat }}$ up the remainder of the channel.

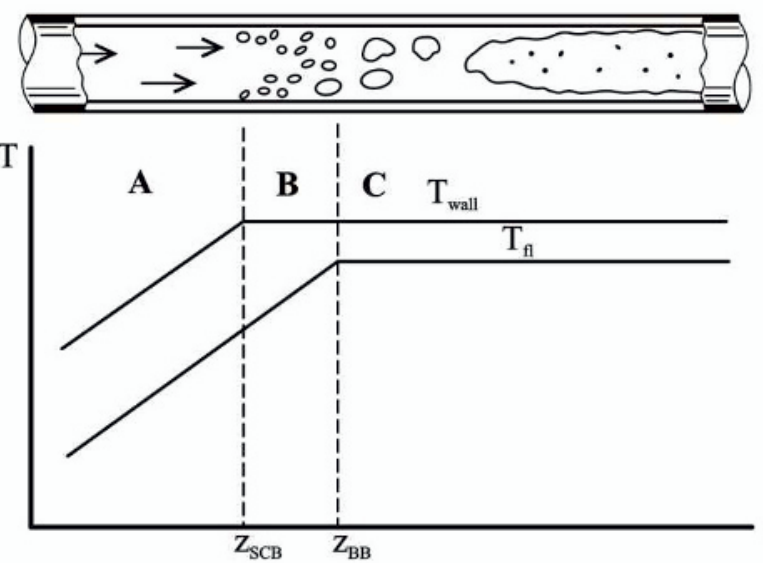

Figure 2 Boilling channel temperature profiles

\section{Two-Phase Flow}

When the coolant is a two-phase mixture of liquid and vapor, the coolant flow pattern can become quite complicated. One can distinguish several types of two-phase flow, as show in figure 3. It is useful to introduce some definitions characterizing the vapor content of a coolant at rest:

$$
\begin{gathered}
\text { static quality } \equiv x_{s} \equiv \frac{\text { mass of vapor in mixture }}{\text { total mass of mixture }} \\
\text { void fraction } \equiv \alpha \equiv \\
\text { volume of vapor in mixture } \\
\text { total volume of liquid - vapor mixture }
\end{gathered}
$$

We can compute these quantities in terms of the cross-sectional areas in the channels occupied by liquid $\left(A_{l}\right)$ and vapor $\left(A_{g}\right)$. The void fraction is given by

$$
\alpha=\frac{A_{g}}{A_{g}+A_{l}}
$$

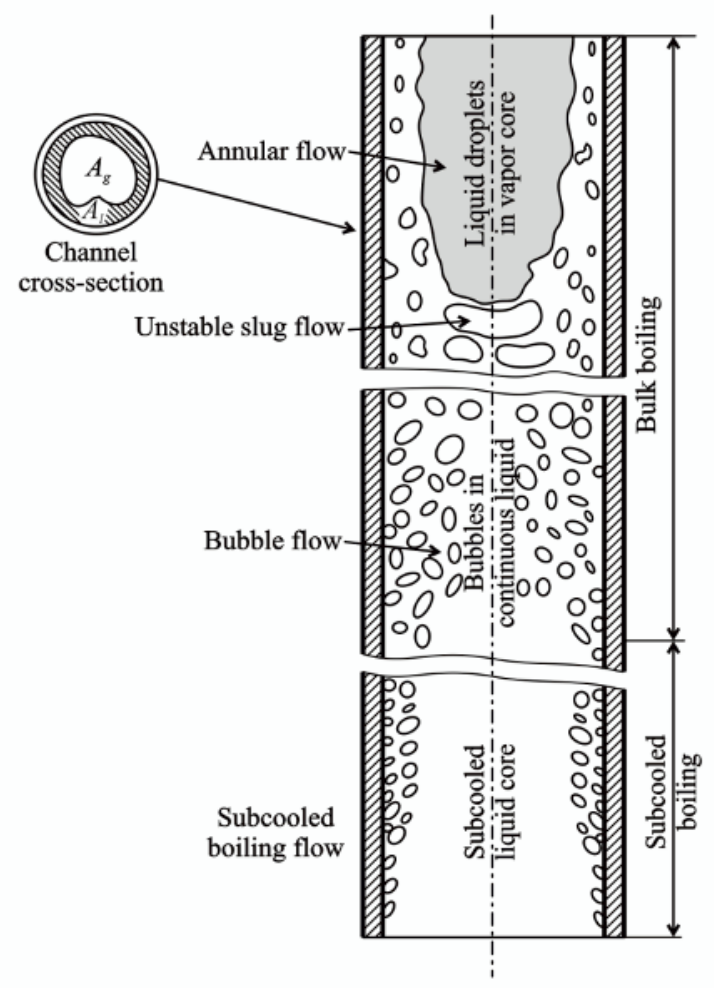

Figure 3 Flow patterns in a heated channel

while the static quality is

$$
\begin{array}{r}
x_{s}=\frac{\rho_{g} \cdot A_{g}}{\rho_{g} \cdot A_{g}+\rho_{l} \cdot A_{l}}=\frac{1}{1+\frac{\rho_{l} \cdot A_{l}}{\rho_{g} \cdot A_{g}}}= \\
=\frac{1}{1+\frac{\rho_{l}}{\rho_{g}}\left(\frac{1-\alpha}{\alpha}\right)}
\end{array}
$$

For example, the void fraction is given for water at various qualities and pressures in figure 4 .

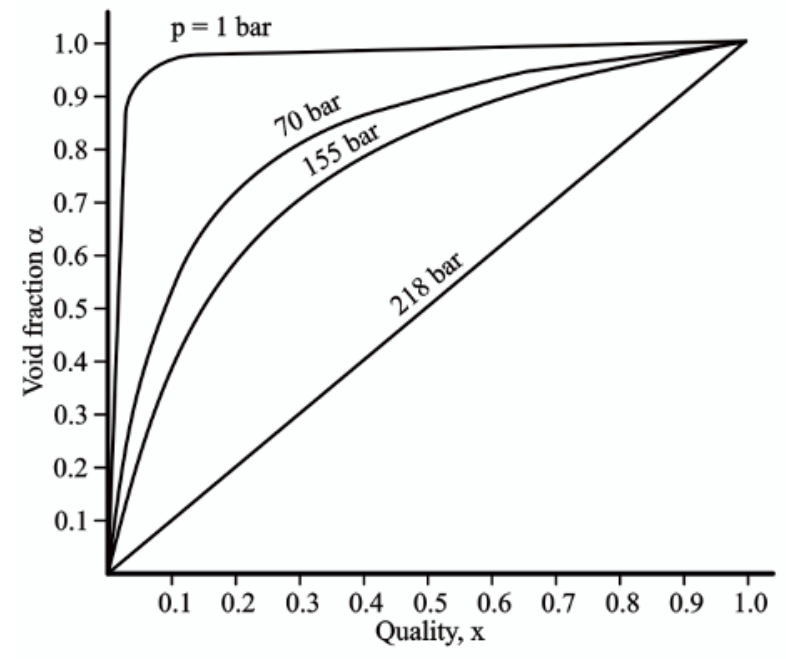

Figure 4 Void fraction versus quality for bulk boiling 
Table 1 Thermodynamic Data on Steam

\begin{tabular}{|c|c|c|c|c|}
\hline $\mathrm{p}$ (bar) & $\mathrm{T}_{\text {sat }}\left[{ }^{\circ} \mathrm{C}\right]$ & $\mathrm{h}_{\mathrm{f}}[\mathrm{MJ} / \mathrm{kg}]$ & $\mathrm{h}_{\mathrm{fg}}[\mathrm{MJ} / \mathrm{kg}]$ & \\
\hline 1 & 100 & 0.419 & 2.26 & \\
\hline 70 & 285 & 1.26 & 1.51 & \\
\hline 155 & 345 & 1.68 & 0.96 & \\
\hline 218 & 373 & 2.10 & 0 & \\
\hline $\mathrm{p}$ (bar) & $c_{p}$ & $\chi_{\mathrm{s}}$ & $\rho_{\mathrm{l}} / \rho_{\mathrm{g}}$ & $\alpha$ \\
\hline 1 & 4.186 & 0.1 & 1602 & 0.995 \\
\hline 70 & 5.44 & 0.1 & 20.6 & 0.7 \\
\hline 155 & 7.96 & 0.1 & 5.9 & 0.4 \\
\hline 218 & $\sim 62$ & 0.1 & 1 & 0.1 \\
\hline
\end{tabular}

When the two-phase mixture is flowing, we must be more careful in our definitions since the vapor has a tendency to slip past the liquid because of its buoyancy. We then must define the quality in terms of a parameter $S$ known as the slip ratio which relates the different flow velocities:

$$
\text { slip ratio } \equiv S \equiv \frac{c_{g}}{c_{l}}
$$

Then we can define a flow quality

$$
\begin{gathered}
x \equiv \frac{\text { mass flow rate of vapor }}{\text { total mass flow rate }}=\frac{w_{g}}{w_{g}+w_{l}} \\
x=\frac{\rho_{g} \cdot A_{g} \cdot c_{g}}{\rho_{g} \cdot A_{g} \cdot c_{g}+\rho_{l} \cdot A_{l} \cdot c_{l}}=\frac{1}{1+\frac{\rho_{l} \cdot A_{l} \cdot c_{l}}{\rho_{g} \cdot A_{g} \cdot c_{g}}}
\end{gathered}
$$

or

$$
x=\frac{1}{1+\frac{\rho_{l}}{\rho_{g}} \cdot\left(\frac{1-\alpha}{\alpha}\right) \cdot \frac{1}{S}}
$$

One can invert this to write the void fraction $\alpha$ as

$$
\alpha=\frac{1}{1+\frac{\rho_{g}}{\rho_{l}} \cdot\left(\frac{1-x}{x}\right) \cdot S}
$$

This latter equation is sometimes known as the "S $-\alpha-x$ " relationship.

\section{Description of Flow Patterns}

When two phases flow co-currently in a channel, they can arrange themselves in a number of different configurations, called flow patterns or flow regimes. Each flow pattern is characterized by a relatively similar distribution of the two phases and of their interfaces. Transition from one flow pattern to another takes place whenever a major change occurs in geometry of the gas-liquid interface. Figure 5 shows the flow patterns observed in a vertical circular duct, and figure 6 illustrates typical flow regimes reported for horizontal flow in a pipe. These include:

Bublle flow ... The liquid is the continuous phase and the gas is dispersed in the liquid in the form of bubbles of variable shape and size.

Slug flow ... is characterized by large gas bubbles almost filling the channel and separated by slugs of liquid.

Churn flow ... transition state between Slug and Annular flow.

Annular flow ... consists of an annular liquid film and of a gas core with or without drops of liquid in it.
Spray, mist, dispersed or fog flow ... The gas occupies most of the cross-sectional area and the liquid is in the form of small droplets dispersed in the gas.

Stratified flow ... Because of gravity forces the liquid flows along the bottom of a horizontal or inclined channel, and the gas flows above it.

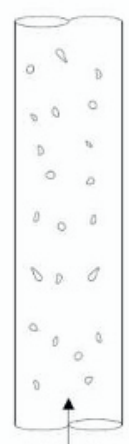

Bubble flow

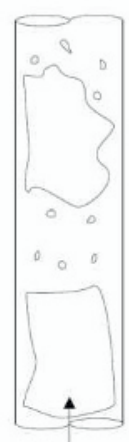

Slug or plug flow

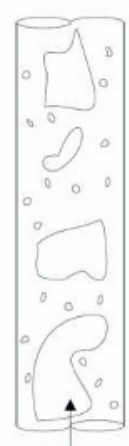

Churn

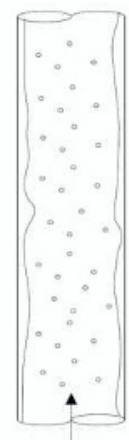

Annular flow

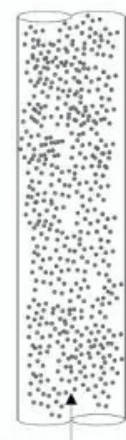

Dispersed flow
Figure 5 Flow patterns in the vertical direction

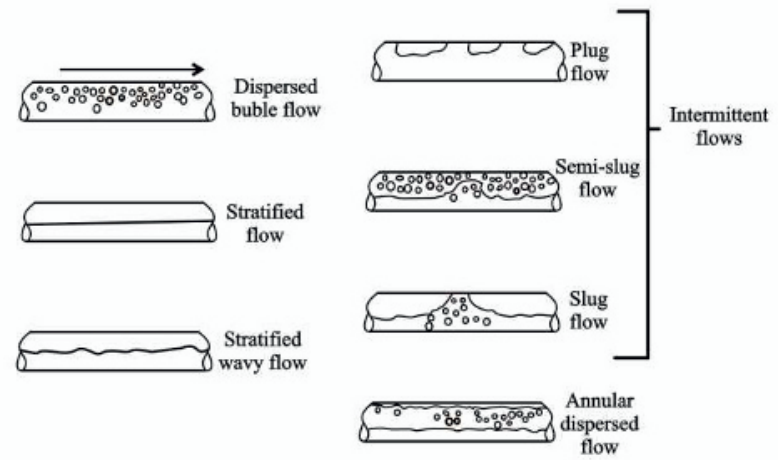

Figure 6 Flow patterns in the horizontal direction

A variety of other flow regimes have been reported in the literature, some of which are illustrated in figure 5 and figure 6 . In many cases, new names have been introduced to better define the distribution of the two phases.

\section{Map of Flow Regimes}

\subsection{Hewitt and Roberts}

Axis $\mathrm{x}$ :

$$
\rho_{l}\left\{j_{l}\right\}^{2}=\frac{G_{m}^{2} \cdot(1-x)^{2}}{\rho_{l}}
$$

Axis y:

$$
\rho_{g}\left\{j_{g}\right\}^{2}=\frac{G_{m}^{2} \cdot x^{2}}{\rho_{g}}
$$

Dispersed bubbles flow regime will be in:

Axis $\mathrm{x}$ :

$$
\rho_{l}\left\{j_{l}\right\}^{2}\left[\frac{\mathrm{kg}}{\mathrm{s}^{2} \cdot \mathrm{m}}\right] \geq 3 \cdot 10^{3}
$$

Axis y:

$$
\rho_{v}\left\{j_{v}\right\}^{2}\left[\frac{\mathrm{kg}}{\mathrm{s}^{2} \cdot \mathrm{m}}\right] \geq 10^{2}
$$




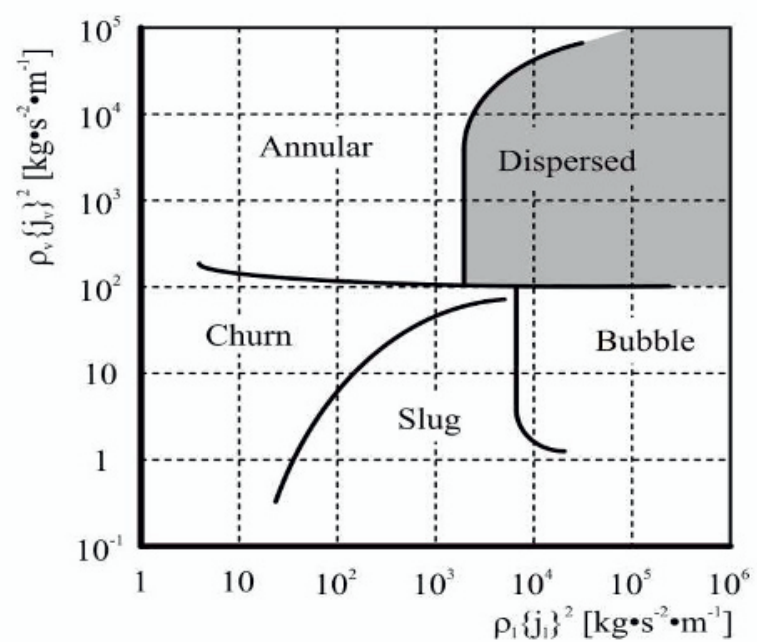

Figure 7 Distribution of the types of a two-phase flow (Hewitt \& Roberts)

where indicated in Hewitt and Roberts are:

$$
\begin{gathered}
G_{m}^{2}=\left(\frac{\dot{m}_{g}+m_{l}}{A}\right)^{2}\left[\frac{\mathrm{kg}}{\mathrm{s}^{2} \cdot \mathrm{m}}\right] \\
j_{i}=\frac{\dot{m}_{i}}{\rho_{l} \cdot A}=\frac{\dot{V}_{i}}{A}\left[\frac{\frac{\mathrm{kg}}{\mathrm{s}}}{\frac{\mathrm{kg}}{\mathrm{m}^{3}} \cdot \mathrm{m}^{2}}=\frac{\mathrm{m}}{\mathrm{s}}\right]
\end{gathered}
$$

where:

$$
\begin{array}{lll}
\dot{V}_{i} & {\left[\mathrm{~m}^{3} / \mathrm{s}\right]} & \text { volumetric flow } \\
\dot{m}_{i} & {[\mathrm{~kg} / \mathrm{s}]} & \text { mass flow } \\
j & {[\mathrm{~m} / \mathrm{s}]} & \text { superficial velocit } \\
\text { flow area relative to the whole su } \\
A & {\left[\mathrm{~m}^{2}\right]} & \text { flow area } \\
i & \text { index } & i=l, g \\
l & \text { index } & \text { liquid } \\
g & \text { index } & \text { gas } \\
x & {[1]} & \text { flow quality }
\end{array}
$$$$
j \quad[\mathrm{~m} / \mathrm{s}] \quad \text { superficial velocity (flow rate of } \mathrm{m}^{2}
$$$$
\text { flow area relative to the whole surface of pipes A) }
$$

$G_{m} \quad\left[\mathrm{~kg} /\left(\mathrm{s} \cdot \mathrm{m}^{2}\right)\right]$ mass flow in flow area

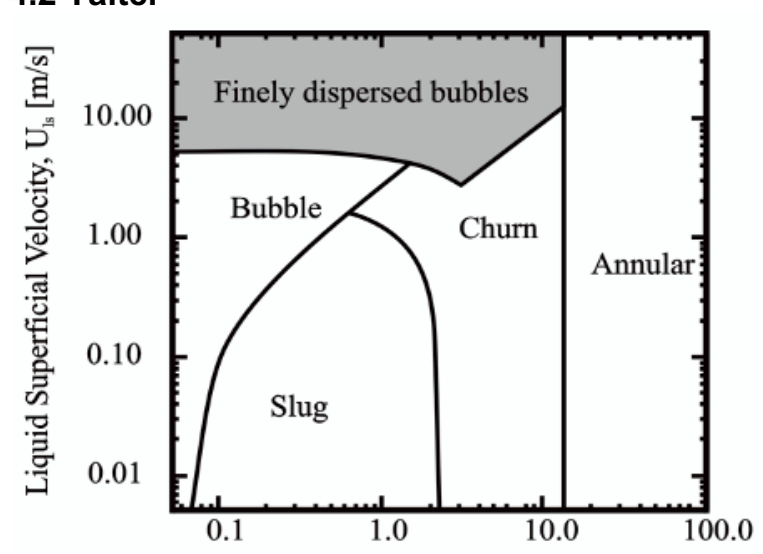

$$
\begin{gathered}
x=\frac{\dot{m}_{g}}{\dot{m}_{g}+\dot{m}_{l}} \\
G_{m}=G_{l}+G_{g} \\
G_{m} \cdot x=G_{g} \\
G_{m} \cdot(1-x)=G_{l}
\end{gathered}
$$

Gas Superficial Velocity, $U_{g g}[\mathrm{~m} / \mathrm{s}]$

Figure 8 Map of distribution of the types of a two-phase flow (Taitel) [1]

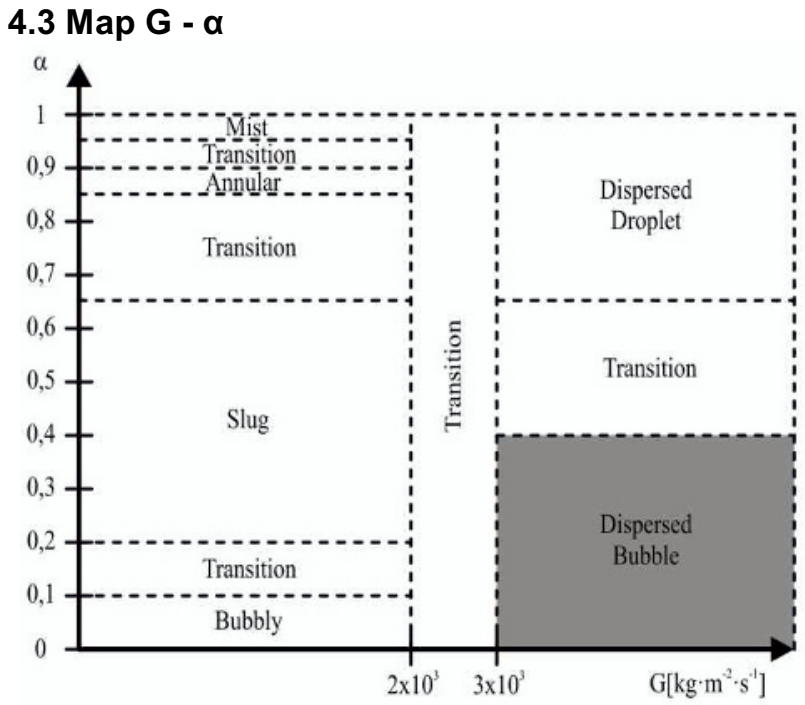

Figure 9 RELAP 5

\subsubsection{Determination of the parameter $\alpha$}

HEM model

$$
\begin{gathered}
s=1, \quad c_{g}=c_{l}, \quad h_{m}^{+}=h \\
\Rightarrow \quad \alpha=\frac{1}{1+\frac{1-x}{x} \cdot \frac{\rho_{g}}{\rho_{f}} \cdot 1}, x=\frac{h-h_{f}}{h_{g}-h_{f}}
\end{gathered}
$$

Drift Flux model

$$
c_{g} \neq c_{l}, s=?
$$

Thermodynamic equilibrium. The model is derived in Zuber, Findlay - Journal of Heat Transfer, where was introduced shifting velocity $c_{g j}$ (drift velocity) so that local velocity vapor (gas):

$$
\begin{aligned}
& \begin{array}{c}
c_{g}=j+c_{g j} \rightarrow \text { shift of } c_{g j} \text { to } j \text { see }(\mathbf{1 6}) \\
\text { is derived: }
\end{array} \\
& \{\alpha\}=\frac{1}{C_{0} \cdot\left(1+\frac{1-x}{x} \cdot \frac{\rho_{g}}{\rho_{l}}\right)+\frac{C_{g j} \cdot \rho_{l}}{x \cdot G_{m}}}, \\
& \quad s=C_{0} \cdot+\frac{C_{0}-1}{1-x} \cdot \frac{x \cdot \rho_{l}}{\rho_{g}}+\frac{c_{g j} \cdot \rho_{l}}{x \cdot G_{m}}
\end{aligned}
$$

where $C_{0}=\frac{\left\{\alpha_{j}\right\}}{\{\alpha\}\{j\}}$ is called the distribution parameter which expresses the distribution of the steam (or gas) content on the surface (for uniform distribution $C_{0}=1$ ). Is necessary to know $C_{0}, c_{g j}$. The latest set of parameters $C_{0}, c_{g j}$ can be found in $[14,18]$.

\section{Dispersion Bubble Regime}

Dispersion bubble regime occurs when turbulence shear forces are equal to or larger than the buoyancy force in the fluid.

Buoyancy force:

$$
F_{\text {buoy }}=\alpha_{g} \cdot g \cdot\left(\rho_{l}-\rho_{g}\right) \cdot A_{s} \cdot D_{h}
$$

Strength of turbulence

$$
\begin{aligned}
F_{\text {tur }}= & C_{l v} \cdot A_{s} \cdot \rho_{l} \cdot \frac{c_{l}^{2}}{2} \cdot\left(1-\alpha_{g}\right) \\
& F_{\text {buoy }} \leq F_{\text {turb }} \Longrightarrow c_{l} \geq c_{l \text { krit }}
\end{aligned}
$$


Critical fluid velocity $c_{l \text { crit disp bubble for transition to }}$ dispersed bubble flow can be determined from a comparison of forces.

$$
\begin{aligned}
& c_{l \text { crit disp bubble }} \\
& =\sqrt{\frac{2 \cdot \alpha_{g} \cdot g \cdot\left(\rho_{l}-\rho_{g}\right) \cdot A_{s} \cdot D_{h}}{\left(1-\alpha_{g}\right) \cdot C_{l v} \cdot A_{s} \cdot \rho_{l}}}
\end{aligned}
$$

where:

$\begin{array}{lll}D_{h} & {[\mathrm{~m}]} & \text { hydraulic diameter pipe } \\ A_{s} & {\left[\mathrm{~m}^{2}\right]} & \text { flow area } A_{s}=\frac{\pi \cdot D_{h}}{4} \\ g & {\left[\mathrm{~m} / \mathrm{s}^{2}\right]} & \text { acceleration of gravity } \\ \rho & {\left[\mathrm{kg} / \mathrm{m}^{3}\right]} & \text { density } \\ l & \text { index } & \text { liquid } \\ g & \text { index } & \text { gas } \\ C_{l v} & {[1]} & \text { drag coefficient of liquid }\end{array}$

For $C_{l v}$ we use the empirical relationship

$$
C_{l v}=\frac{0.046}{\sqrt{\frac{\rho_{l} \cdot c_{l} \cdot D_{h}}{\eta_{l}}}}=\frac{0.046}{\sqrt{\frac{4 \cdot W_{l}}{\pi \cdot D_{h}^{2} \cdot \eta_{l}}}}
$$

$c_{l} \quad[\mathrm{~m} / \mathrm{s}] \quad$ velocity of liquid

$W_{l}[\mathrm{~kg} / \mathrm{s}] \quad$ mass flow rate

These relationships apply for $\alpha_{g} \leq 0.56$ and a vertical pipe.

\section{Conditions for the Use of "Gas-Lift"}

Specification of the parameters of the core must be based on the requirements for the performance of MSR. The performance MSR will be used for production of electricity for technological purposes, or in combination. The volume of the core using the center determines specific power $q$.

a) natural convection $q \approx 10\left[\mathrm{MW} / \mathrm{m}^{3}\right]$

b) "gas-lift" $\quad q \approx(25 \div 40)\left[\mathrm{MW} / \mathrm{m}^{3}\right]$

c) forced convection $q \approx 50\left[\mathrm{MW} / \mathrm{m}^{3}\right]$

Average tensile chimney $D$ determined from the criticality condition $\left(k_{e f}<0.95\right)$. We will consider an infinitely tall cylinder surrounded by a graphite reflector. Dimension $D$ can be adjusted (while maintaining subcriticality) according to the flow velocity.

It is necessary to structurally arrange the primary circuit of the reactor and our "Two-phase flow demonstrator" (TFD), so that the total pressure losses were minimal. Effectiveness of natural flow and "gas-lift" is strongly dependent.

\section{Procedure to Calculate the Flow}

We obtain this relation for natural heat flowing in the primary circuit - on condition that the temperature changes only in the active zone and the heat exchanger from the solution of the continuity equation and the Bernoulli's equation:

$$
\tilde{\chi}_{p r}=\frac{\chi_{p r}}{\bar{A}}
$$

where

$\chi_{p r}$ are total hydraulic losses in the primary circuit $\bar{A} \quad$ is the medium flow cross-section of the circuit
It applies :

$$
\begin{gathered}
\frac{1}{\bar{A}}=\frac{\bar{\rho}}{L} \cdot \int_{x_{0}}^{x_{L}} \frac{d x}{\rho(x) \cdot A(x)} \\
\bar{\rho}=\frac{1}{V} \cdot \int_{x_{0}}^{x_{L}} \rho(x) \cdot A(x) \cdot d x
\end{gathered}
$$

where :

$\bar{\rho} \quad$ is the medium density of the coolant in the circuit

$$
W_{\infty}=\left[\frac{2 \cdot \bar{\rho}^{2} \cdot \beta \cdot g \cdot P}{\tilde{\chi}_{p r} \cdot c_{p}} \cdot\left(\bar{z}_{E}-\bar{z}_{C}\right)\right]^{1 / 3}
$$

where $P[\mathrm{~W}]$ is the total heat output of the zone $W_{l}=\rho_{l}\left(T_{\max }\right) \cdot A_{k} \cdot c_{v l}$ and $W_{g}=\rho_{g}\left(T_{g}\right) \cdot A_{k} \cdot c_{v g}$ where the cross-sectional area of the tractive "chimney" is $A_{k}=\pi \cdot D^{2} / 4, \rho$ is density, index $l$ is the fluid, $g$ is the gas, $W_{\infty}[\mathrm{kg} / \mathrm{s}]$ is the weight flow rate.

$$
W_{\infty}^{\text {gas-lift }}=W_{l}+W_{g}\left[\frac{k g}{s}\right]
$$

Using $W_{\infty}^{\text {gas-lift }}$ it is necessary to use in general $c_{p}$ and $\rho$ for a two-phase mixture and simultaneously determine the new losses, because $W_{\infty}^{\text {gas-lift }}$ increases the flow velocity in the whole primary circuit.

The amount of the gas supplied by the $\mathrm{m}$ vents into the tractive cylinder of the gas-lift $W_{g}[\mathrm{~kg} / \mathrm{s}]$ is determined by the following relation from [3].

$$
\begin{array}{r}
W_{g}=C_{d} \cdot \frac{\pi}{4} \cdot d^{2} \cdot m \cdot p_{1} \cdot \sqrt{\frac{1}{r \cdot T_{g}}-\frac{2 \cdot \kappa}{\kappa-1}} . \\
\cdot \sqrt{\left(\frac{p_{2}}{p_{1}}\right)^{2 / \kappa}-\left(\frac{p_{2}}{p_{1}}\right)^{\kappa-1 / \kappa}}
\end{array}
$$

where

$m$ [1] is the number of holes with a diameter $\mathrm{d}$, $C_{d}$ [1] is the loss factor, which must be determined experimentally,

$p_{1} \quad[P a] \quad$ is the pressure of gas before the throttle hole,

$p_{2} \quad[P a] \quad$ is the pressure of gas after the throttle hole,

$\kappa=1,66$

$\kappa=1,4$

$\kappa=1,33$

$\kappa=1,3$

$r=\frac{R}{M}\left[\frac{J}{k g \cdot K}\right]$

$R \quad\left[\frac{\mathrm{J}}{\mathrm{kmol} \cdot \mathrm{K}}\right]$ is the universal gas constant,

$M \quad\left[\frac{\mathrm{kg}}{\mathrm{mol}}\right] \quad$ the mass of one mole of gas

Changes and flow properties at various temperatures and pressures of the gas and the liquid in tension and chimney losses in two-phase flow in the entire circuit are described in [20].

Homogeneous mixture of liquid and gas density $\rho_{h}$ varies speed $c_{h}$. Modeling of two-phase flow forecast includes a two-stage friction factor and the properties of the mixture.

Bertil and Whalley (1982) [21] recommended use Colebrook equation for friction properties using a twophase Reynolds numbers listed for the homogeneous model, which describes the current phase of medium properties of individual phases. 
The Colebrook equation is implicit in the two-phase friction factor and its determination requires an iterative approach. In 1983 S.E.Heeland determine approximate relationship for the two-phase friction factor, which differs from the equation Colebrook to $2 \%$ and does not require iteration.

$$
\frac{1}{\dot{f}_{T P}}=-1.8 \cdot \log _{10}\left[\frac{6.9}{R e_{T P}}+\left(\frac{\frac{\varepsilon_{R}}{D}}{3.7}\right)^{1.11}\right]
$$

where

$\dot{f}_{T P}$ two-phase friction factor

$\varepsilon_{R} \quad$ wall roughness

$$
\rho_{T P}=\rho_{g} \cdot \varepsilon_{f h}+\rho_{l} \cdot\left(1-\varepsilon_{f h}\right)
$$

where $\rho_{T P}$ is density two-phase mixture in the pipe the gas lift.

Two-phase Reynolds number

$$
R e_{T P}=\frac{\left(\rho_{g} \cdot c_{g}+\rho_{l} \cdot c_{l}\right)}{\eta_{g h}} \cdot D_{h}
$$

Two-phase dynamic viscosity $\eta$ is determined by the experimental relationship

$$
\eta_{T P}=\eta_{g} \cdot \varepsilon_{g h}+\eta_{l} \cdot\left(1-\varepsilon_{g h}\right) \cdot\left(1+2.5 \cdot \varepsilon_{g h}\right)
$$

The overall average speed homogeneous mixture can be easily determined

$$
c_{h}=\left(\frac{W_{l}}{\rho_{l}}+\frac{W_{g}}{\rho_{g}}\right) \cdot \frac{1}{A}
$$

\section{Effect of Temperature and Pressure on the Flow}

We consider that we have a bubble of radius $R$ filled with gas at pressure $p_{2 g}$ and temperature $T_{g 1}$. It is located in the local fluid pressure $p_{1}(0)$ and temperature $T_{1}$. Bubble moves towards the liquid relative velocity $c_{b}$. Usually is $T_{1}>T_{g 1}$ and $p_{1}>p_{g 2}$.

We determine the amount of heat that goes from liquid to bubble in time $d t$.

$$
\frac{d Q}{d t}=\pi \cdot D^{2} \cdot \alpha \cdot \Delta T
$$

For outer wrap ball says Whitaker (1972) [22] universal correlation valid for forced flow and use that to measure the heat transfer to the bubbles.

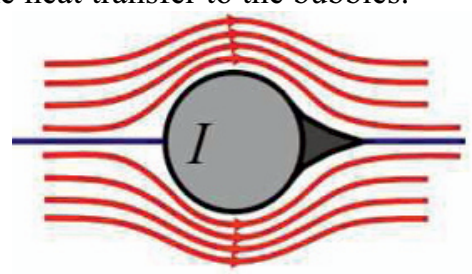

\section{Figure 10}

$3.5 \leq \operatorname{Re} \leq 7.6 \cdot 10^{4}$

$0.71 \leq \operatorname{Pr} \leq 380$

$\mathrm{Nu}=2+\left(0.4 \cdot \operatorname{Re}^{1 / 4}+0.06 \cdot \operatorname{Re}^{2 / 3}\right) \operatorname{Pr}^{0.4}(\eta / \eta w)^{1 / 4}$

Member of $\mathrm{Re}^{1 / 2}$ corresponds to the heat transfer in laminar boundary layer; $\mathrm{Re}^{2 / 3}$ corresponds to the heat transfer in wake. Correction for variable uses dynamic viscosity coefficient 0.25 , which is used by more than 0.14 coefficient of relationship Sieder-Tate. For $\mathrm{Re} \rightarrow 0, \mathrm{Nu}$ approaching asymptotic value of 2 (corresponding to heat transfer in the upright environment) ie molecular heat transfer.

$$
\alpha=\frac{N u \cdot \lambda_{1}}{D} \quad\left[\frac{W}{m^{2} \cdot K}\right]
$$

For true balance bubble $p_{g}>p_{1}$, by which we determine the initial bubble size $R_{0}$

$$
\left(p_{g}-p_{1}\right)=\frac{4 \cdot \sigma_{l}}{d}=\frac{2 \cdot \sigma_{l}}{R_{0}}
$$

where $\sigma_{l}$ is surface tension of the liquid.

From (33) we determine the amount of heat that goes into the bubble from the surrounding fluid. At an initial temperature $T_{g 1}$ of the gas bubble energy

$$
Q_{0}=\rho_{g} \cdot c_{g v} \cdot V \cdot T_{g l}[\mathrm{~J}]
$$

where

$c_{g v}$ is specific heat of gas at constant volume

$V \quad$ is the volume of gas in the bubble

Divide the height of the chimney tensile $K$ intervals $\Delta h_{k}$, where $k \in\langle 1, K\rangle$. In time $\Delta t_{1}=\Delta h_{k} / c_{b l}$ goes into heat $\Delta Q_{1}$ bubbles that increase the gas temperature $\Delta T_{g}=T_{g 2}-T_{g 1}$.

For a general interval $k$ applies:

$$
\begin{gathered}
\Delta Q_{k}=\rho_{g k}\left(\bar{T}_{k}\right) \cdot c_{g v} \cdot \bar{V}_{k} \cdot\left(T_{g(k+1)}-T_{g k}\right) \\
\Delta Q_{k}=\alpha\left(\bar{T}_{k}\right) \cdot 4 \cdot R^{2} \cdot\left(T_{l k}-T_{g k}\right) \cdot \Delta t_{k}
\end{gathered}
$$

and thence:

$$
\begin{gathered}
T_{g(k+1)}=\left[\frac{\alpha\left(\bar{T}_{k}\right) \cdot 4 \cdot R^{2} \cdot\left(T_{l k}-T_{g k}\right) \cdot \Delta t_{k}}{\rho_{g k}\left(\bar{T}_{k}\right) \cdot c_{g v} \cdot \bar{V}_{k}}\right. \\
\left.+T_{g k}\right]
\end{gathered}
$$

For the calculation must be finely dividing the flow path in tension channel and iteratively determine the increase in flow velocity and the growth rate of losses.

Besides the heat transfer to the bubble we have to consider the influence of changes in pressure and flow velocity of the liquid in the pressure with a height of chimney.

Next, we determine the size of the bubbles, depending on the pressure and temperature of the liquid velocity in tension chimney.

We expect:

Above the surface of liquid gas pressure $p_{\text {sys }}$.

The point $z=0$ is the pressure in the fluid flow velocity with consideration equal to:

$$
\begin{gathered}
p_{l}(0)=p_{\text {sys }}+\rho_{l}\left(T_{l}(0)\right) \cdot g \cdot(H+\Delta H-z) \\
-\rho_{l}\left(T_{l}(0)\right) \cdot \frac{c_{l}^{2}}{2}
\end{gathered}
$$

where

$H \quad$ is the height of the chimney

$\Delta H \quad$ is the water level in the pressurizer

For height $z$

$$
\begin{gathered}
p_{l}(z)=p_{\text {sys }}+\rho_{l}\left(T_{l}(z)\right) \cdot g \cdot(H+\Delta H-z) \\
-\rho_{l}\left(T_{l}(z)\right) \cdot \frac{c_{l}^{2}}{2}
\end{gathered}
$$

Bubble size is determined by a balance of forces on the surface of the bubbles. For $z=0$ 
$4 \cdot \pi \cdot R^{2} \cdot p_{g}(0)=4 \cdot \pi \cdot R^{2} \cdot\left(p_{l}(0)+\frac{2 \cdot \sigma}{R}\right)$

The radius of the bubble

$$
\begin{gathered}
R(0)=\frac{2 \cdot \sigma}{p_{g}(0)-p_{l}(0)} \\
p_{l}(0)=\left\{p_{g h}+\rho_{l}\left(T_{l}(0)\right) \cdot g \cdot(H+\Delta H)\right\} \\
+\rho_{l}\left(T_{l}(0)\right) \cdot \frac{c_{l}^{2}(0)}{2} \\
R(z)=\frac{2 \cdot \sigma\left(T_{l}(z)\right)}{p_{g}(z)-p_{l}(z)} \\
p_{l}(z)=\left\{p_{g h}+\rho_{l}\left(T_{l}(z)\right) \cdot g \cdot(H+\Delta H-z)\right\} \\
+\rho_{l}\left(T_{l}(z)\right) \cdot \frac{c_{l}^{2}(z)}{2}
\end{gathered}
$$

The bubble will grow with the increasing influence of pressure reduction in tension in the liquid chimney and heat transfer from the liquid to the gas in the bubble. This further increases the lifting force and the mass flow quantity $W_{\infty}^{\text {gas-lift }}$, ie speed mixture flow, but also the losses in the circuit.

If we consider the distribution of tensile chimney on volumes $V_{k}=A \cdot \Delta h_{k}(z)$ and the assumption of medium-sized bubbles (where we identify $R_{k}$ according to $(45)$ we can determine the number of bubbles $N_{k}$ in the volume $V_{k}$.

$$
N_{k}=\frac{V_{k} \cdot\left(1-\varepsilon_{l}\right)}{\frac{4}{3} \cdot \pi \cdot R_{k}^{3}}
$$

The considerations (36) and (37) we know the amount of heat transferred into one bubble in step $k Q_{r k}$. Of the $N_{k} Q_{r k}$ know the amount of heat removed fluid

$$
\Delta Q_{l k}=\bar{c} \cdot p_{l} \cdot W_{k} \cdot\left[T_{l k}-T_{l(k-1)}\right]
$$

\section{Note:}

The molecular surface energy is then characterized by variable $\omega$ the product of the surface tension $\sigma$ and values $V_{m}^{2 / 3}$ where $V_{m}=M / \rho$ is the mol volume.

$$
\omega=\sigma \cdot V_{m}^{2 / 3}=\sigma \cdot\left(\frac{M}{\rho}\right)^{2 / 3}
$$

Expressing molar surface energy is not calculated with a geometric factor $(36 \cdot \pi)^{1 / 3}$, which is the same for all liquids.

The dependence of $\omega(t)$ on the temperature $t\left[{ }^{\circ} \mathrm{C}\right]$ determine Eötvös (1886) empirical relationship [22]:

$$
\omega(\mathrm{t})=\sigma(t) \cdot\left(\frac{M}{\rho(t)}\right)^{2 / 3}=K \cdot\left[t_{k r}-(t+\sigma)\right]
$$

where:

$k \quad$ constant of proportionality

$t_{k r} \quad$ critical temperature of the liquid

Knowing the value of the surface tension $\sigma\left(t_{0}\right)$ for temperature $t_{0}$, then using equation $(\mathbf{5 0})$ we determine the constant of proportionality $k$ and then when knowledge of temperature $t_{k}$ and density $\rho_{k}$ to easily determine $\sigma(t)$.

\section{Description of the experimental device}

The research of two-phase flow was the KKE assembled experimental device on which to plan further treatment.

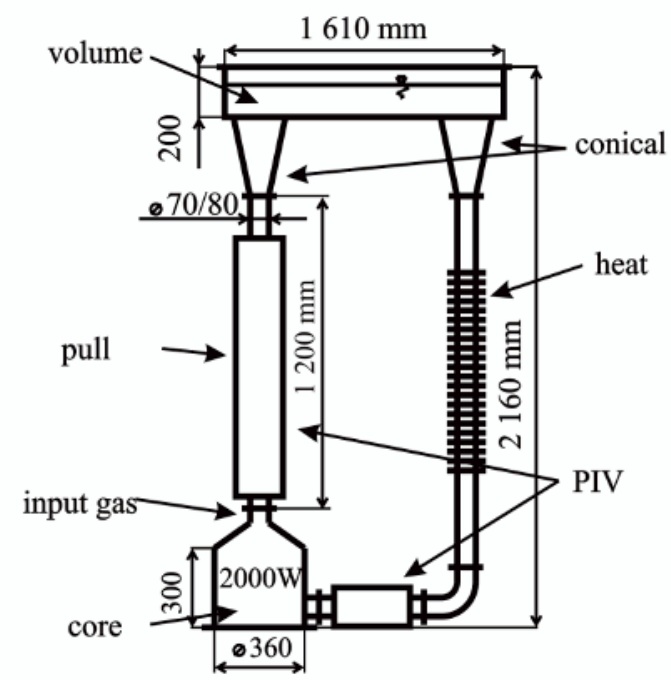

Figure 11 The model of the two-phase flow demonstrator

First, we set the corresponding diagram of experimental apparatus MSR primary circuit high in integral structure.

The experimental device consists of the series of construction parts that have been named after the parts of the primary circuit that they represent. The model of the whole device including the description of its main parts is shown in the figure 11.

There were used two types of the construction material - metal from the carbonaceous steel and plexiglass. The most parts are welded from the $2 \mathrm{~mm}$ thick metal plates. The remaining parts that have to be transparent in order to perform the PIV measurements are made from the $5 \mathrm{~mm}$ or $10 \mathrm{~mm}$ thick plexiglass depending on the strain of the part. The maximum dimensions of the device are $2160 \times 1100 \times 360 \mathrm{~mm}$ (height $\mathrm{x}$ length $\mathrm{x}$ width).

The active zone is represented here by the cylindrical metal container of $360 \mathrm{~mm}$ diameter and height of 300 $\mathrm{mm}$. There is a conic crossing in its upper part and they are of air intake for the gas-lift follows. Gas phase must be separated from liquid. The centrifugal separator is for this purpose. Its aerodynamic design is provided according [24].The heat source in the model core secures a $2000 \mathrm{~W}$ heating unit that is bolted and correctly sealed to the wall of the container in its bottom part in order not to obstruct the flowing water and therefore not to increase the pressure losses. The bottom of the core, that has to be removable in order of assembling and potential exchange of the heating unit, is connected with the cylindrical part of the core using the flange and twelve screws. There is bolted a ball valve for filling and drainage in the bottom most part of the core. 


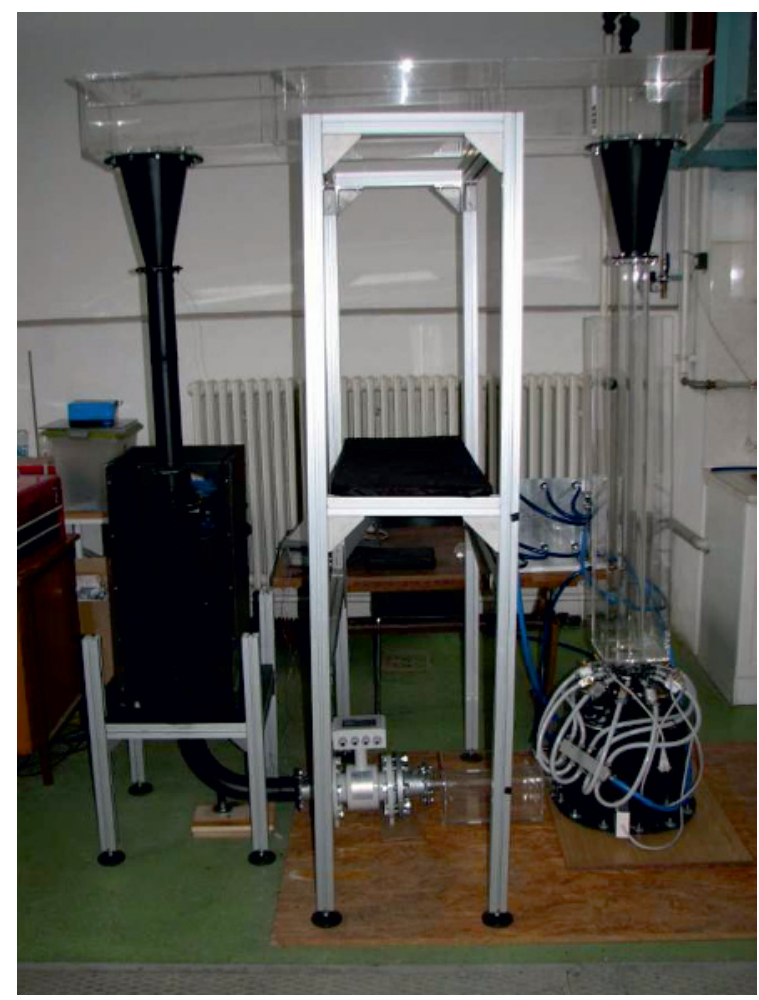

Figure 12 Two-phase flow demonstrator

\section{Conclusions}

Description of formulas for calculations and designs for gas-lift reactors also serve gas lift model in the laboratory of the Department of Power System Engineering. The model in the first stage is used as media for water and air dispersion modeling using bubble type two-phase flow, which is in terms of the operation of nuclear reactors advantageous. Measurements on the model will be compared with the method Chexal - Lellouche [14]. In addition, there is a comparison of experimental data with numerical simulations.

Chexal-Lellouche (1996) Void Fraction Model was based on the model of Zuber and Findlay (1965) and summarizes the possibilities of two-phase flow models for cases with water vapor, water, air and liquids used in refrigeration technology. Model describes the flow of vertical, horizontal and angled. In these 3 cases were examined hundreds of experiments performed in the world and EPRI. Comparison of experiments with computations show the match into a difference of $\pm 10 \%$. Chexal-Lellouche model has been implemented in emergency programs for computing the number of nuclear power plants relapse MOD3 5, (also MOD3.1 and 3.2.1.2) and RETRAN-3D MOD002.

Method Chexal-Lellouche [14] serves also study the possibility of errors when using other media, which are not experimentally proven, such as the use of gas-lift with the use He of high temperature MSR based fluoride salts. Preparing the comparison model and works based on similarity theory.

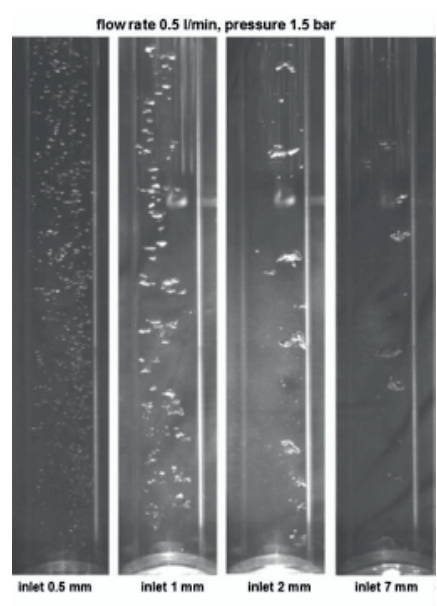

Figure 13

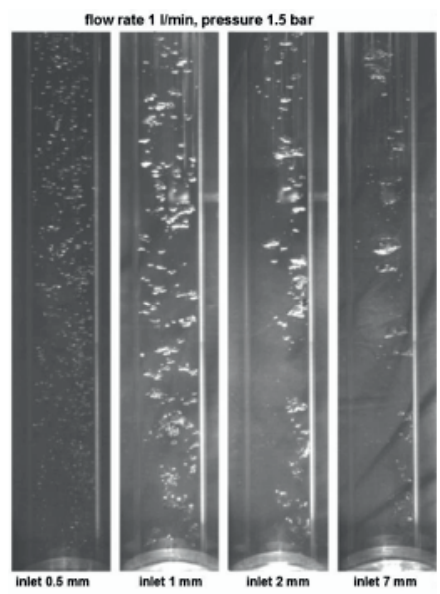

Figure 14

\section{Acknowledgements}

The presented research is supported by the project CZ.1.05/2.1.00/03.0108 (Sustainable Energy, SUSEN) and student project SGS-2012-072 (Zvyšování účinnosti, spolehlivosti a životnosti energetických strojů a zařízení 2).

\section{References}

1. S. Levy: Two-Phase Flow in Complex Systems. (John Wiley \& Sons, 1999)

2. G. Takacz: Gas-lift Manual, (Penn Well, 2003)

3. V. Valenta, research report KKE 03/2009

4. V. Valenta, research report KKE 01-12

5. V. Valenta, research report KKE 05/ 2012

6. V. Valenta, research report KKE O4/2010.

7. V. Valenta, research report KKE 02/ 2011.

8. S. J. White: Bubble Pump Design and Performance. (Georgia Institut of Technology, 2001)

9. W.J. Moore: Fyzikální chemie (SNTL Praha, 1979)

10. D.R.H. Bertie, P.B. Walley, Journal of Multiphase Flow 8 (1982)

11. Y. Taitel, D. Borner, A.E. Buckler, AICHE Journal 26 (1980)

12. S.N.Šrin: Sdilení tepla (SNTL Praha, 1968) 
13. V. Valenta, research report KKE 02-12

14. B. Chexal, M. Merilo, J. Maulbetch, J. Horowitz, J. Harrison, J. Westacott, C. Peterson, W. Kastner, H. Schmidt, TR-106326 (1997)

15. V. Valenta, Modelování a měření v energetice (2010)

16. V. Valenta, research report TECH 03/2002

17. V. Valenta, research report TECH 02/2005

18. K. Kawanishi, Y. Hirao, A. Tsuge, Nuclear Engineering and Design 120, (1990)

19. J.J. Duderstadt, L.J. Hamilton, Nuclear Reactor Analysis. (John Wiley and Sons, 1976)

20. V. Valenta, P. Žitek, research report KKE 04/2012

21. D.R.H. Bertie, P.B. Walley, Journal of Multiphase Flow 8 (1982)

22. V. Kellö, A. Tkáč: Fyzikálna chémia. (Alfa Bratislava 1969)

23. A.C. Hoffmann, L.E. Stein, Gas Cyclones and Swirl Tubes, (Springer, 2008)

24. J. Polanský, R. Gášpár, ASME Mechanical Engineering Congress and Exposition 7, part A Fluids and Heat Transfer (2012) 